\title{
The UHF oscillators based on a HEMT structure with negative conductivity
}

\author{
Andriy Semenov, Olena Semenova \\ Faculty of Radio Engineering, Telecommunication and \\ Instrument Engineering \\ Vinnytsia National Technical University \\ Vinnytsia, Ukraine \\ Semenov79@ukr.net
}

\begin{abstract}
The article considers a possibility of constructing microwave oscillators on a transistor structure with negative conductivity based on two HEMT. Obtained non-linear equations can be used to model microwave oscillator parameters and characteristics with an error not more than $10 \%$.
\end{abstract}

Keywords-ocsilator; HEMT; negative conductivity; transistor structure

\section{INTRODUCTION}

UHF (ultra high frequency) voltage-controlled oscillators are widely used in radio engineering and electronics. Their construction can be considerably simplified using impedance properties of a transistor structure with negative conductivity. In such case control voltage changes oscillation frequency in limits $(3,0 . .9,5) \%$ at small non-linear distortions of harmonic oscillations. HEMT utilization allows increasing oscillation frequency in the voltage-controlled oscillator (VCO) and considerable decreasing the total harmonic distortion (THD $<0,297 \%)$. The urgent problem of the oscillator projecting is the transistor structure based on the HEMT I/V curve approximation problem.

\section{OSCILLATOR ON THE TWO-ELECTRODE HEMT STRUCTURE}

The UHF oscillator on the two-electrode HEMT structure is shown on fig.1. The obtained equation of the two-electrode HEMT structure I/V curve is expressed by (1). The equation of the differential conductivity is expressed by (2). Their diagrams are shown on fig. 2.

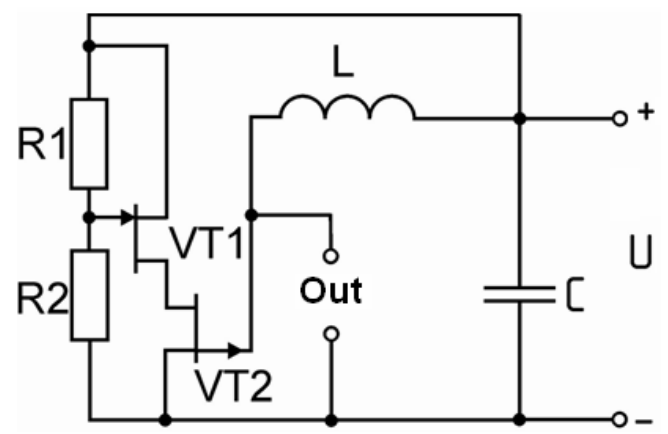

Fig. 1. The electric circuit of the UHF oscillator on the two-electrode HEMT structure

\author{
Oleksandr Osadchuk \\ Faculty of Radio Engineering, Telecommunication and \\ Instrument Engineering \\ Vinnytsia National Technical University \\ Vinnytsia, Ukraine \\ Osadchuk69@mail.ru
}

$I(U)=\frac{U}{R_{1}+R_{2}}+\frac{I_{S 0}}{\tanh M}\left(1-\frac{U}{2 U_{0}} \cdot \frac{R_{1}}{R_{1}+R_{2}}\right)^{2} \tanh \left[M \frac{U / 2 U_{0}}{1-\frac{U}{2 U_{0}} \cdot \frac{R_{1}}{R_{1}+R_{2}}}\right]$

$$
G_{\sim}^{(-)}(U)=\frac{d I}{d U}=\frac{1}{R_{1}+R_{2}}-\frac{I_{S 0}}{2 U_{0}}\left(\frac{2 R_{1}}{R_{1}+R_{2}}\left(1-\frac{U}{2 U_{0}} \cdot \frac{R_{1}}{R_{1}+R_{2}}\right) \times\right.
$$

$\left.\times(\tanh M)^{-1} \tanh \left[M \frac{U / 2 U_{0}}{1-\frac{U}{2 U_{0}} \cdot \frac{R_{1}}{R_{1}+R_{2}}}\right]-\grave{I} \cdot \cosh ^{-2}\left[M \frac{U / 2 U_{0}}{1-\frac{U}{2 U_{0}} \cdot \frac{R_{1}}{R_{1}+R_{2}}}\right]\right)$

where $I_{S 0}$ is the source current at $U_{g d}=0, U_{s d}=U_{0}$; $U_{g d}, U_{s d}$ are the voltages on the gate-drain and source-drain electrodes respectively; $U_{0}$ is the cutoff voltage [1].

The parameter of $M$ is evaluated from the equation $M=S_{\max } \frac{U_{0}}{I_{S 0}}$, where $S_{\max }=\frac{d I_{s}}{d U_{s d}}$ is the drain characteristics conductance of a FET at $U_{s d}=U_{g d}=0[1]$.

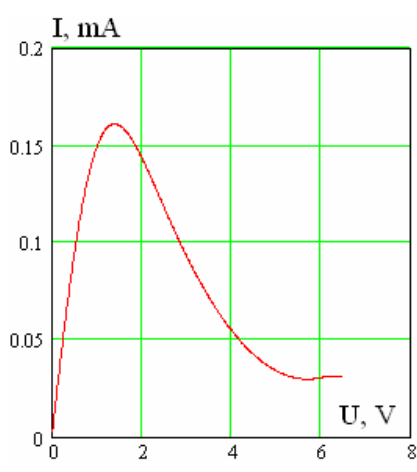

a)

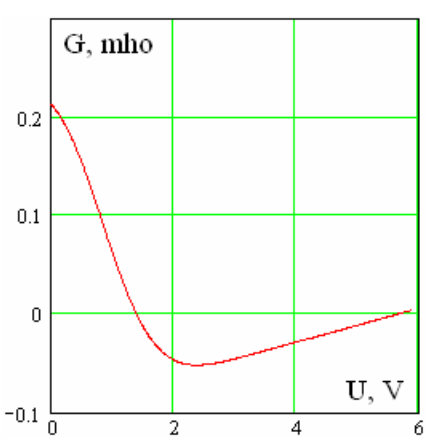

b)
Fig. 2. The approximated $\mathrm{I} / \mathrm{V}$ curve (a) and differential conductivity dependences (b) of the two-electrode HEMT structure

The generated current equation is 
$i_{\grave{O}}(u)=\left[\frac{1}{R_{1}+R_{2}}+\frac{I_{S 0}}{U_{0}} \frac{M}{\tanh M}\left[\frac{1}{2}+\frac{U^{2}}{8 U_{0}^{2}}\left(\left(\frac{R_{1}}{R_{1}+R_{2}}\right)^{2}-\frac{1}{3} M^{2}\right)\right] \times\right.$

$\times U \cos \tau+\ldots$

Second harmonic is on a level of $-53 \mathrm{~dB}$, others are less.

The amplitude balance differential equation is

$$
\begin{aligned}
& T \frac{d U}{d T}=\left(\frac{R_{e q}}{R_{1}+R_{2}}+\frac{I_{S 0} R_{e q}}{2 U_{0}} \frac{M}{\tanh M}-1\right) U+ \\
& +I_{S 0} R_{e q} \frac{M}{\tanh M} \frac{U^{3}}{8 U_{0}^{3}}\left(\left(\frac{R_{1}}{R_{1}+R_{2}}\right)^{2}-\frac{1}{3} M^{2}\right) .
\end{aligned}
$$

The oscillator autoexcitation condition is

$$
\frac{R_{e q}}{R_{1}+R_{2}}+\frac{I_{C 0} R_{e q}}{2 U_{0}} \frac{M}{\tanh M}>1
$$

The oscillator stationary vibration amplitude is

$$
U_{C T}=\frac{2 U_{0} \sqrt{1-\frac{R_{e q}}{R_{1}+R_{2}}-\frac{I_{S 0} R_{e q}}{2 U_{0}} \frac{M}{\tanh M}}}{\sqrt{\frac{I_{C 0} R_{e q}}{2 U_{0}} \frac{M}{\tanh M}\left(\left(\frac{R_{1}}{R_{1}+R_{2}}\right)^{2}-\frac{1}{3} M^{2}\right)}}
$$

Dependence of the oscillation amplitude $U$ on time (at the generation establishing stage)

$$
U(t)=\frac{U(0) a^{\gamma / t}}{\sqrt{1+\left(U^{2}(0) / U_{C T}^{2}\right)(\exp 2 \gamma t-1)}},
$$

where $U(0)$ is the oscillation initial amplitude,

$$
\gamma=\left(\frac{R_{e q}}{R_{1}+R_{2}}+\frac{I_{C 0} R_{e q}}{2 U_{0}} \frac{M}{\tanh M}-1\right) / T
$$

III. OSCILLATOR ON THE THREE-ELECTRODE HEMT STRUCTURE

The electric circuit of the UHF oscillator on the threeelectrode HEMT structure is shown on fig. 3 [2].

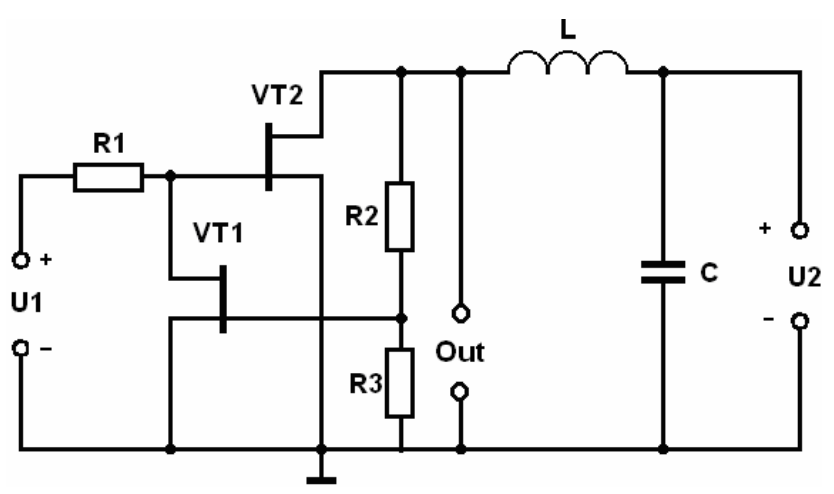

Fig. 3. The electric circuit of the UHF oscillator on the HEMT structure
The experimental I/V curve of the three-electrode HEMT structure is shown on fig. 4 [3].

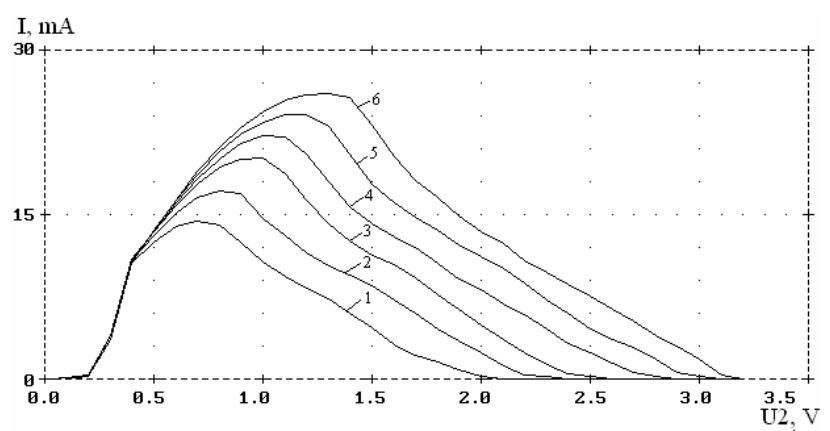

Fig. 4. The I/V curve of the three-electrode HEMT structure of the UHF oscillator at: 1) $\mathrm{U} 1=0,2 \mathrm{~V}$; 2) $\mathrm{U} 1=0,25 \mathrm{~V}$; 3) $\mathrm{U} 1=0,3 \mathrm{~V}$; 4) $\mathrm{U} 1=0,35 \mathrm{~V}$; 5) $\mathrm{U} 1=0,4 \mathrm{~V}$; 6) $\mathrm{U} 1=0.45 \mathrm{~V}$

The equation of the three-electrode HEMT structure I/V curve is expressed by (9). The equation of its negative conductivity is expressed by (10)

$$
\begin{aligned}
& I\left(U_{1}, U_{2}\right)=\frac{U_{2}}{R_{2}+R_{3}}+I_{\tilde{N} 0}\left(1-P 1\left(U_{1}, U_{2}\right)\right)^{2} \times \\
& \times(\tanh M)^{-1} \operatorname{th}\left[M \frac{U_{2} / U_{0}}{1-P 1\left(U_{1}, U_{2}\right)}\right],
\end{aligned}
$$

where

$$
\begin{gathered}
P 1\left(U_{1}, U_{2}\right)=\frac{U_{1}}{U_{0}}-S R_{1}\left[\frac{U_{2}}{U_{0}} \frac{R_{3}}{R_{2}+R_{3}}-\frac{b U_{2}^{2}}{U_{0}}\left(\frac{R_{3}}{R_{2}+R_{3}}\right)^{2}-1\right] . \\
G_{\sim}^{(-)}\left(U_{1}, U_{2}\right)=\frac{\partial I}{\partial U_{2}}=\frac{1}{R 2+R 3}+\frac{I_{S 0}}{U_{0}}(\tanh M)^{-1}\left[1-P 1\left(U_{1}, U_{2}\right)\right] \times \\
\times\left\{2 \cdot S \cdot R 1 \cdot\left[\frac{R 3}{R 2+R 3}-2 b U_{2}\left(\frac{R 3}{R 2+R 3}\right)^{2}\right] \cdot \tanh \left(M \frac{U_{2} / U_{0}}{1-P 1\left(U_{1}, U_{2}\right)}\right)+\right. \\
\left.+\frac{M\left[1-P 1\left(U_{1}, U_{2}\right)\right]}{\cosh ^{2}\left[M \frac{U_{2} / U_{0}}{1-P 1\left(U_{1}, U_{2}\right)}\right]} \cdot \frac{2\left[1-P 1\left(U_{1}, U_{2}\right)\right]-S \cdot R 1-1}{\left[1-P 1\left(U_{1}, U_{2}\right)\right]^{2}}\right\} .
\end{gathered}
$$

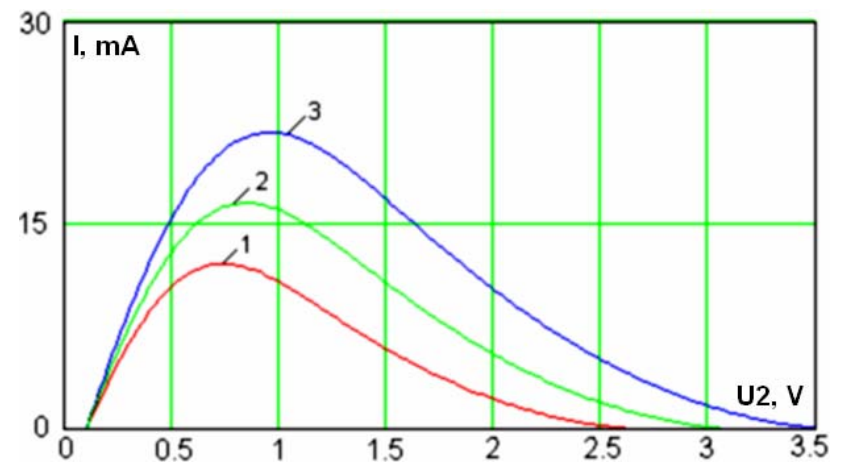

Fig. 5. Approximated static $\mathrm{I} / \mathrm{V}$ curves of the three-electrode HEMT structure at 1) $\mathrm{U}=0,2 \mathrm{~V}$; 2) $\mathrm{U} 1=0,3 \mathrm{~V}$; 3) $\mathrm{U} 1=0,4 \mathrm{~V}$ 


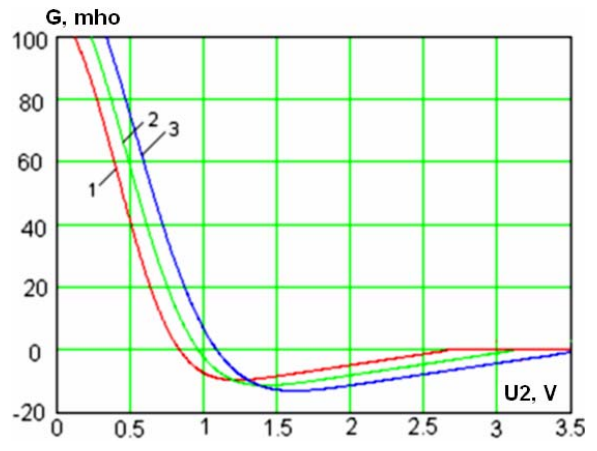

Fig. 6. Diagrams of the differential conductivity of the three-electrode HEMT structure at 1) $\mathrm{U} 1=0,2 \mathrm{~V}$; 2) $\mathrm{U} 1=0,3 \mathrm{~V}$; 3) $\mathrm{U} 1=0,4 \mathrm{~V}$

The generated current equation is

$i_{\dot{O}}(u)=\left[\frac{1}{R_{2}+R_{3}}+\frac{I_{\tilde{N} 0}}{U_{0}} \frac{M}{\tanh M}\left(P_{2}-\frac{U^{2}}{U_{0}^{2}}\left(\frac{R_{3}}{R_{2}+R_{3}}\right)^{2} P_{3}\right)\right] U \cos \tau+\ldots$

where

$$
\begin{gathered}
P_{2}=\left(1+S R_{1}+S^{2} R_{1}^{2}\right)^{2}-3 S R ; \\
P_{3}=\left(1+S R_{1}+S^{2} R_{1}^{2}\right)\left(S^{2} R_{1}^{2}-2 b U_{0}\left(1+S R_{1}\right)\right)+S R_{1}\left(b U_{0}-1\right) .
\end{gathered}
$$

The amplitude balance differential equation is

$$
\begin{gathered}
T \frac{d U}{d t}=\left(\frac{R_{e q}}{R_{2}+R_{3}}+\frac{I_{C 0} R}{U_{0}} \frac{M}{t h M} P_{2}-1\right) U- \\
-I_{C 0} R \frac{M}{\tanh M} \frac{U^{3}}{U_{0}^{3}} P_{3}\left(\frac{R_{3}}{R_{2}+R_{3}}\right)^{2} .
\end{gathered}
$$

The oscillator autoexcitation condition is

$$
\frac{R_{e q}}{R_{2}+R_{3}}+\frac{I_{S 0} R_{e q}}{U_{0}} \frac{M}{\tanh M} P_{2}>1
$$

The oscillator stationary vibration amplitude is

$$
U_{C T}=U_{0} \frac{\sqrt{\frac{R_{e q}}{R_{2}+R_{3}}+\frac{I_{C 0} R_{e q}}{U_{0}} \frac{M}{\tanh M} P_{2}-1}}{\sqrt{\frac{M}{\tanh M} \frac{I_{C 0} R_{e q}}{U_{0}} P_{3}\left(\frac{R_{3}}{R_{2}+R_{3}}\right)^{2}}} .
$$

Dependence of the oscillation amplitude $\mathrm{U}$ on time (at the generation establishing stage)

$$
U(t)=U(0) \exp (\gamma t) / \sqrt{1+\left(U_{0}^{2} / U_{C T}^{2}\right)(\exp 2 \gamma t-1)},
$$

where $U(0)$ is the oscillation initial amplitude,

$$
\gamma=\left(\frac{R_{e q}}{R_{1}+R_{2}}+\frac{I_{C 0} R_{e q}}{U_{0}} \frac{M}{\tanh M} P 2-1\right) / T .
$$

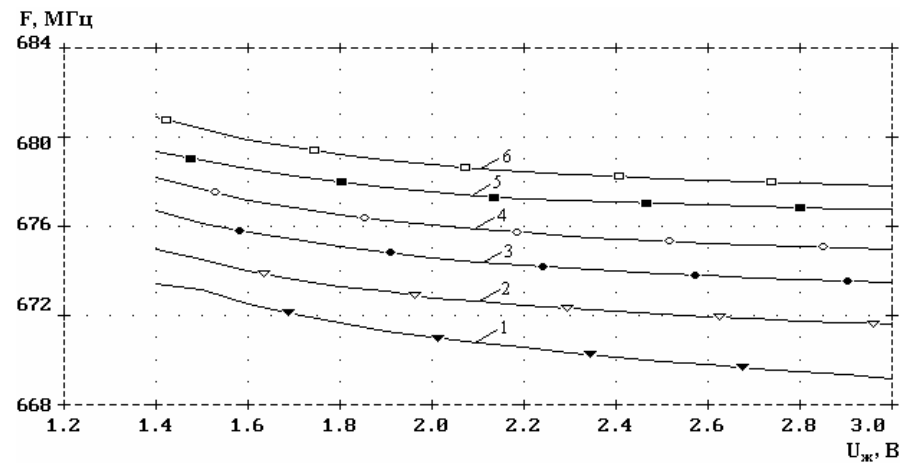

Fig. 7. Experimental dependences of the oscillation frequency change at 1) $\mathrm{U} 1=0,2 \mathrm{~V}$; 2) $\mathrm{U} 1=0,25 \mathrm{~V}$; 3) $\mathrm{U} 1=0,3 \mathrm{~V}$; 4) $\mathrm{U} 1=0,35 \mathrm{~V}$; 5) $\mathrm{U} 1=0,4 \mathrm{~V}$; 6 ) $\mathrm{U} 1=0,45 \mathrm{~V}$

\section{OPTIC-CONTROLLED OSCILLATOR ON THE HEMT STRUCTURE}

The diagram of the optic-controlled oscillator on the HEMP structure is shown on fig. 8 .

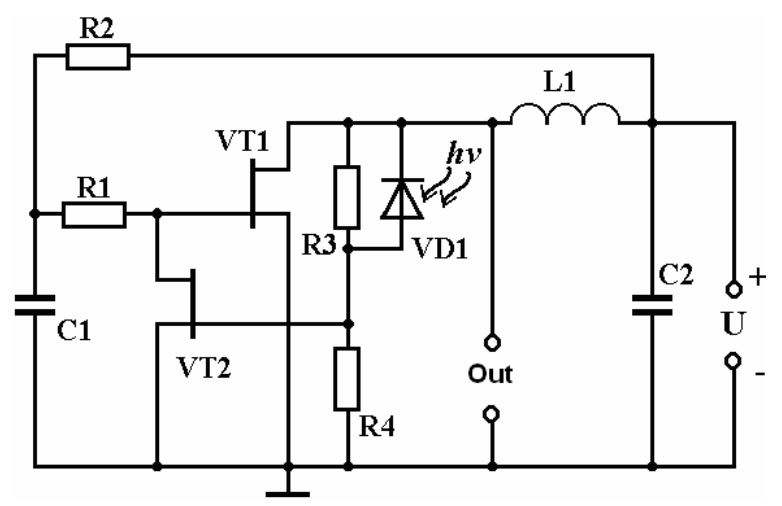

Fig. 8. The electric circuit of the UHF optic-controlled oscillator on the HEMT structure

In the UHF range the HEMT source current is a sum of two components, they are $2 \mathrm{D}$ electron gas and $\mathrm{AlGaAs}$ electrons. Each of them may be expressed as [4]

$$
I_{C i}=q Z n_{S i}\left(U_{g d}, U_{i}(x)\right) v_{i}(x), i=1,2,
$$

where $Z$ is a gate width; $q$ is a electron charge module; $v_{i}$ is a electron drift rate; $U_{i}$ is a model parameter. The drift rate dependence on field strength allowing saturation effect is

$$
v_{i}(x)= \begin{cases}\frac{\mu_{i} E(x)}{1+E(x) / E_{H i}}, & E(x)<E_{H i} ; \\ V_{H i}, & E(x) \geq E_{H i} ;\end{cases}
$$

where $\mu_{i}$ is a mobility in weak field; $E_{H i}$ is a critical electric field when the saturation takes place; $V_{H i}$ is the saturation electron rate at $E_{i}=E_{H i}$.

The HEMT source currents in linear and saturation modes are expressed (21) add (22) respectively 


$$
\begin{aligned}
& I_{\tilde{n} \ddot{e},}=\frac{A_{i}}{L+U_{\tilde{n} \ddot{e}} / A i_{3}} \times \\
& \times\left[B_{i} U_{\tilde{n} \tilde{e}}-\ln \cosh \frac{U_{g d}-U_{m i}-U_{\tilde{n} \ddot{e}}}{U_{1 i}}+\ln \cosh \frac{U_{g d}-U_{m i}}{U_{1 i}}\right] ; \\
& { }_{\tilde{n} i,}=\frac{\grave{A}_{3}}{L i_{3}+U i_{3} / A i_{3}} \times \\
& \times\left[B_{i} U_{\tilde{n} \tilde{e}}-\ln \cosh \frac{U_{g d}-U_{m i}-U_{\tilde{n} \tilde{e}}}{U_{1 i}}+\ln \cosh \frac{U_{g d}-U_{m i}}{U_{1 i}}\right],
\end{aligned}
$$

where

$$
A_{i}=q \mu_{i} Z n_{0 i}\left(1-\alpha_{i}\right) U_{l i} B_{i}=\alpha_{i} /\left(1-\alpha_{i}\right) U_{l i},
$$

$U_{H i}$ is the channel potential in the saturation point, $L_{H}$ is the saturation useful channel length calculated from the equation

$$
L i_{i}=L-\frac{2 d i_{i}}{\pi} \sinh ^{-1} \frac{\pi\left(U_{s d}-U i_{i}\right)}{2 d i_{i} E i_{i}} .
$$

Experimental diagrams of the oscillation frequency sensitivity dependence on the radiating power change are shown on fig. 9.

\section{$\mathrm{F}, \mathrm{MHz}$}

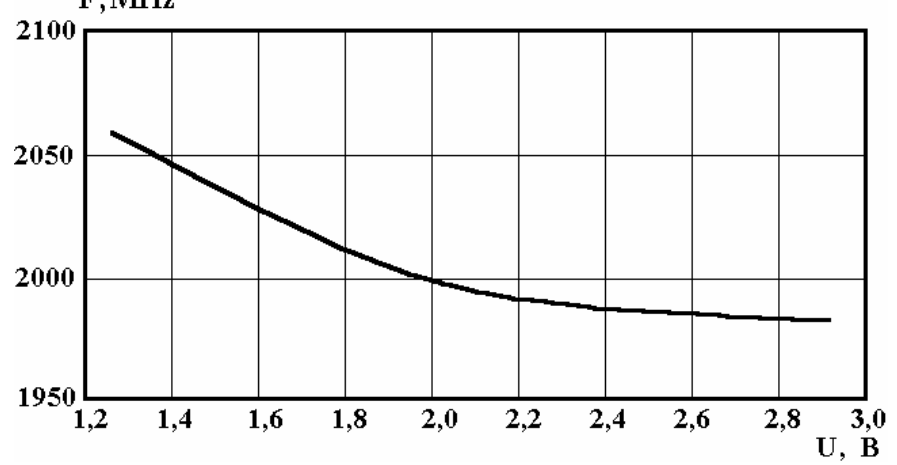

a)

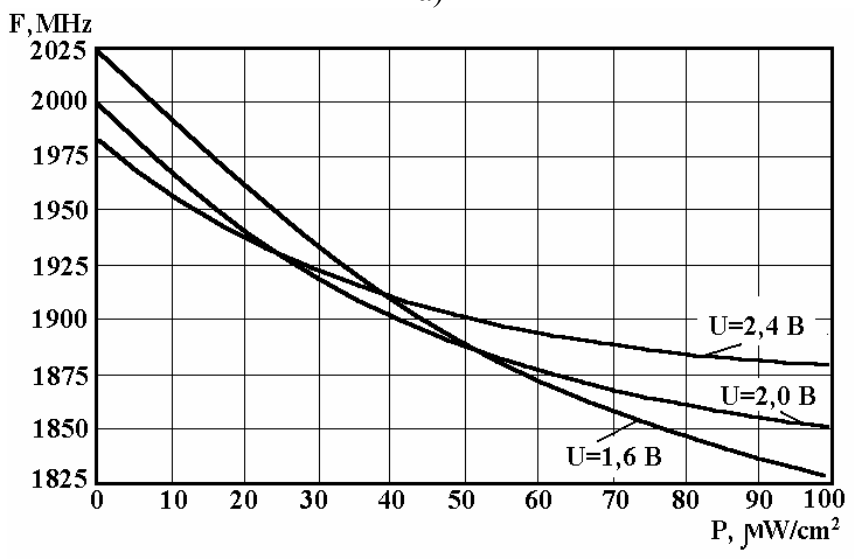

b)

Fig. 9. Diagram of the oscillation frequency change with the supply voltage (a) and radiating power density (b)

The convertion function of radiating power density into the oscillation frequency is obtained

$$
F_{0}=0,1125 \sqrt{\frac{B_{1}+\sqrt{B_{1}^{2}+4 L_{1} C_{h v} R_{d}^{2}\left(P_{h v}\right) \tilde{N}_{d}^{2}\left(P_{h v}\right)}}{L_{1} C_{g s 2} R_{d}^{2}\left(P_{h v}\right) \tilde{N}_{d}^{2}\left(P_{h v}\right)}},
$$

where

$$
B_{1}=R_{d}^{2}\left(P_{h v}\right) \tilde{N}_{d}^{2}\left(P_{h v}\right)+C_{g s 2} R_{d}^{2}\left(P_{h v}\right) \tilde{N}_{d}\left(P_{h v}\right)-L_{1} C_{g s 2} .
$$

From figs. 9, 10 oscillation frequency relative optical tuning is claimed to be $9,88 \%$ while electrical tuning is claimed to be $3,7 \%$.

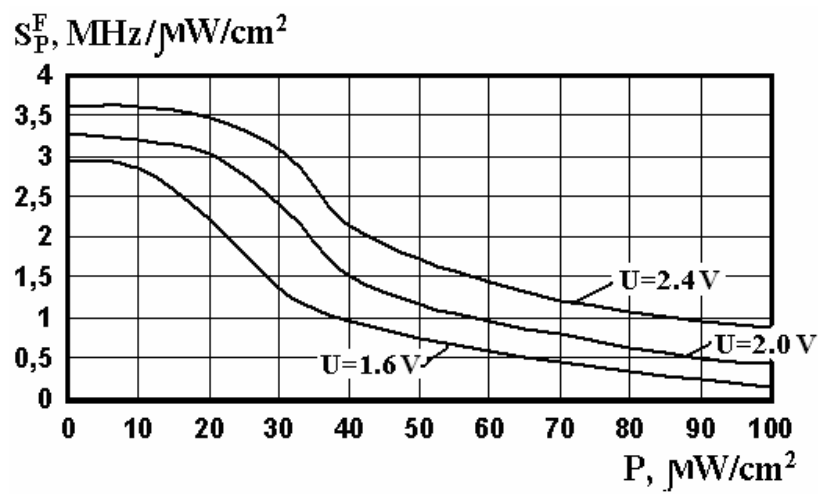

Fig. 10. Dependence of sensitivity of the UHF optic-controlled oscillator on the HEMT structure on the radiating power density at different values ot the supply voltage

\section{REFERENCES}

[1] Molotkov V.I., Potapov E.I. Issledovanie VAKh malomoshchnykh polevykh tranzistorov i lyambda-diodov i raschet amplitud avtogeneratora na lyambda-diode [Study of current-voltage characteristics of low-power field-effect transistors and diodes lambda and the calculation of the amplitudes of the oscillator on the lambda diode]. Radioelektronika. - 1991. Vol. 34, No 11, pp. 108-110.

[2] Leon O. Chua, Juebang Yu, Youying Yu. Bipolar - JFET - MOSFET Negative Resistance Devices// IEEE, Vol.Cas-32. №1, January 1985. P. 46-61.

[3] Angelov I., Rorsman N., Stenarson J. et al. An empirical table-based FET model// IEEE Transactions on Microwave Theory and Technique. 1999. Vol.47, №12. - P. 2350-2356.

[4] Zinkovskyi Iu.F., Koval A.V. Komp'iuterne skhemotekhnichne modeliuvannia elementiv radioelektroniky. Ch. 2 [Computer simulation of electronics circuit elements. Part. 2]. Kyiv. - 2013. - 376 p. 